\title{
LOS PENSADORES DE LA LIBERTAD (o un Universo Escondido Entre Papeles Sueltos)
}

\section{THE THINKERS OF FREEDOM (or a Universe Hidden Between Loose Papers)}

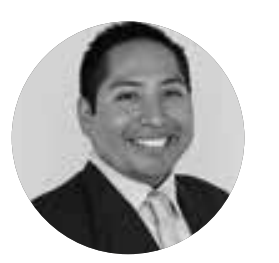

\section{Beltrán Gómez Híjar}

Código ORCID: 0000-0002-5129-2555

Politólogo y magíster en Administración

y Gerencia Social

Lima - Perú

Correo electrónico: beltrangomezhijar@gmail.com

Recibido: Agosto 2020

Aprobado: Setiembre 2020

Cómo citar este artículo: Gómez, B. (2020).

Los pensadores de la libertad-o un universo escondido entre papeles sueltos-

Review of Global Management, 6(1), 07-13.

\section{RESUMEN}

En la historia del pensamiento económico y político hay una diversidad de autores que tienen diferentes interpretaciones de la naturaleza humana, de las instituciones y de cómo se debe organizar la sociedad. Una rama es la que se conforma por los pensadores de la Escuela Austriaca de Economía, que, además de realizar grandes aportes al pensamiento económico, hicieron una incursión trascendental en otras disciplinas, como la filosofía, la ciencia política y el derecho, entre otras. Ellos, a su vez, forman parte de un grupo mayor: el de los filósofos de la libertad, es decir, de aquellos pensadores que defendían intelectualmente la libertad del individuo frente a la coacción arbitraria de un tercero, especialmente del Estado.

Palabras clave: Hayek, Mises, Menger, libertad, socialismo, Escuela Austriaca de Economía.

\section{ABSTRACT}

In the history of economic and political thought, there is a diversity of authors who have different interpretations of human nature, institutions, and of how society should be organized. Among these authors, there is one branch, formed by the thinkers of the Austrian School of Economics, who, in addition to their great contributions to economic thought, also made a transcendental incursion into other disciplines, such as philosophy, political science, law, among others. These thinkers, in turn, are part of a larger group: that of the philosophers of freedom, that is, of those thinkers who intelectually defended individual freedom against arbitrary coercion of a third party, especially the State.

Keywords: Hayek, Mises, Menger, liberty, socialism, Austrian School of Economics 
La biblioteca de la Facultad de Ciencias Sociales era mi lugar favorito de lectura. No era mi facultad, ciertamente, pues yo pertenecía a la Facultad de Derecho y Ciencia Politica, pero aquella biblioteca tenía un encanto especial para la lectura, quizá por la altura de su techo, por encontrarse en el edificio antiguo de la universidad ${ }^{1}$; o porque leer en los escritorios ubicados en el altillo le daban a uno más privacidad, sintiéndote a solas con el autor de turno y su pensamiento. Esto compensaba la molestia de caminar varios metros hasta llegar allá, pasando previamente por una escalera que solía estar a oscuras.

Ahí, recuerdo haber devorado escritos de ciencia política de Giovanni Sartori, Charles Merriam, David Easton, Arend Lijphart, Gabriel Almond, Robert Dahl, Maurice Duverg Vernon Van Dyke, Samuel P. Huntington; de autores provenientes de la sociologia, como Emile Durkheim, Max Weber, de estudiosos de la hlosolita politica, como Norber , Comor Sun Tzu Y muchos otros a que identificarlos explćcitandial largarian demasiado la lista solo empezando por los clásicos de la filosofía política.

Pero en medio de tantos papeles sueltos, viendo en retrospectiva, de vez en cuando (y sin darme cuenta en aguel entonces). aparecía una pequeña ventana de luz que me invitaba a entrar a otro universo, uno cuya existencia desco nocía, y que contenía el pensamiento que defendía la idea de la libertad del hombre frente a la coacción arbitraria de un tercero, principalmente frente al gran leviatán²: el Estado.

${ }^{1}$ El local central de la Universidad Nacional Federico Villarreal (UNFV), perteneció al colegio jesuita de la I nmaculada, ubicado en el centro de Lima, cuya primera piedra fue colocada en 1901, y cuya fachada se terminaria de construir en 1920; la cual todavia se enservay es ll lagar donde hasta hoy se encuentra la biblioteca ceden a la UNFV (Colegio de la Inmaculada Jesuitas - Lima sf 'Monstruo biblico descrito en el libro de Job (41) y que el discurso politico usa para referirse al Estado a través del filósofo Thomas Hobbes, quien titula su gran libro de filosofía politica, Leviatán, o materia, forma y poder de un Estado eclesiástico y civil (1651)
En las separatas del libro, Teoría de la democracia (tomo 2 , Los problemas clásicos). publicado en 1988 del afamado y ya desaparecido politólogo italiano, Giovanni Sartori se menciona en varias oportunidades a Friedrich A. von Hayek. Si Sartori, un autor cuyas obras me habían fascinado lo nombraba más veces que a Hegel, Kelsen, Lenin o Madison, entonces tendria que ser tambien un autor interesante. Decidi ingresar por aquella rendija de luz, a pesar de que nadie me habia pedido leerlo. Empujado por mi curiosidad empece a leer unas líneas de su libro intitulado Los fundamentos de la libertad

Fue con la lectura completa de The Constitution of Liberty5, publicado en 1960, en que descubrí el inicio del hilo de Ariadna ${ }^{6}$. Ese libro era una mezcla de ciencia politica y filosofila politica, escrito por un académico de quien tenia referencias desde el campo de la economía Cuando Hayek mo a civis fasa aue cóf fuertemente ińnicamente hasta ese momento no penśd aue h ten

Detrás de esa afirmación existía una poderosa idea, que atacaba una creencia ya habitual y extendida en la sociedad, la cual nos dice que, si algo anda mal en nuestra realidad, podemos hacer cambios en nuestras instituciones, ahora, principalmente utilizando el derecho, y así, más pronto que tarde, tendremos el paraíso deseado por todos. Pero como sabemos, esa idea suele llevar, en un primer
nivel a intervencionismos estatales puntuales: luego a cambios constitucionales irreflexivos; $y$ finalmente pasa

Sartori tiene una bibliografía vasta que contiene un océano de conocimientos en el cual me sumergi en aquella épocal leyendo sus textos (además del los dos tomos del ya mencionado) Partidos ysistemas de partidos; Homo videns; Elementos de teoria politica; Ingenieria constlucional comparada; La politicalogogica y método ên ${ }^{4}$ En una entrevista realizada por James Buchanan (octubre de 1978 . Hayek calificó este texto como uno de ciencia política. Aquí se concentra su pensamiento filissófico politico de la libertad, vinculándolo con la política del mundo real.

${ }^{-5}$ The Constitution of Luberty se publicó en castellano en 1961, bajo titulo de Los fundamentos de la libertac

adna le entrega un hiloa cer al Minotauro, pueda salir del

- Para Hayek, el constructivismo no es racionalismo y la razón noes antenior a la civilizacion, sino que ambas se desarrollaron coniunta. mente. Ass, de manera crítica, caracteriza al constructivismo diciendo: "Puesto que el hombre ha creado las instituciones de la sociedad y de la civilizacion, puede tambien cambiarlas a discrecion para que a una fase final donde el autoritarismo desplaza a la democracia En esta instancia, el poder se concentra en esfera politica, para desde ahí planificar y poner en marcha la "nueva sociedad", teniendo como resultado un paraíso acaso insostenible, que llega para unos pocos, por no decir solamente para los planificadores estatales y las autoridades mesiánicas.

Un remolino de nuevas concepciones; un conjunto de huevos picos montaniosos, desde dónde ver e interpretar la realidad humana, me fueron ofrecidos con la lectura de aquel libro. ¿Por que no lo había leido antes? Hayek es un autor que merece ser conocido masivamente, pensé. Si bien tuvo un texto que lo volvió una especie de rockstar en un sector de la Academia, Camino de servidumbre ${ }^{8}$ (The Road to Serfdom, en el original), publicado en 1944, en plena II ouerra Mundal, y difundido en gran escala en los EE.UU. por la revista Readers Digest, luego de esta conflagración

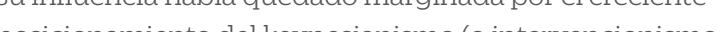
estatal en la economía' ${ }^{10}$ globalmente.

Cuando llegó a mis manos aquel libro su lectura me dejó en profunda reflexión empezando por la dedicatoria Esta dice. "A los socialistas de todos los partidos" : Qué quería decir? ¿Acaso estos no estaban sólo en los partidos que se denominaban socialistas o comunistas? Avido por encontrar las respuestas a estas interrogantes. leíc casi compulsivamente y, rápidamente, pude dar con las respuestas. Efectivamente, el socialismo se había convertido en un pensamiento que, como el éter (que se creía que estaba en todo el universo), se encontraba en la mente de los politicos de todas las tiendas políticas, en mayor 0 menor medida. Un pensamiento unico que, como tal, no acepta ideas que lo contradigan, volviéndose intolerante y acaparador ${ }^{11}$

camino de servidumbre también dice que el nazismo (o el nacional socialismo) no era una rama del capitalismo reaccionario al socialismo, como solian decir los marxistas Ino que ena de la misma especie soclatista, y prueba de ello s que ambos luchaban por obten los votos del mismo sermento poblacional Además

${ }_{8}^{8}$ Publicado en 1944, primero en Inglaterra (marzo) y Australia (julio), y luego en EEUU. (setiembre) por Routledge y University of Chicago Press.

'Hizo una publicación condensada que salió a las calles en 1945, Isto en el momento en que el autor se encontraba atrav ceeano Attantico para dar unas conferenclas

Esta idea es opinión del autor
A partir de 1914 surgen de las filas del socialismo marxista un predicador tras otro que conducen al redil del nacionalsocialismo, no a los conservadores reaccionarios sino a los trabajadores manuales y a la uventud idealista. Sólo más tarde alcanzó la marea del socialismo nacionalista considerable importancia y se

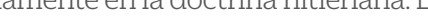
histona belica de 1914... es el comienzo del moderno deschnolviniento que produjo el nacionalsocialismo, elcual surgio durante este periodo gracias en buena parte a la asistencia de viejos socialistas (Hayek, 1944/2017, p. 257)

na idea completamente herética para el pensamiento único.

El pensador del siglo XX que mejor ha condensado y quien más lejos ha llegado a desenredar la madeja de la hea del pensa con ocasión del recibimiento de este galardón, dijo:

El Premio Nobel confiere a un individuo una autoridad que en economía ningún hombre debería poseer (...) hecho una contribución distintiva a la ciencia econó. mica deba ser omnicompetente en todos los problemas de la sociedad (...) Estoy casi inclinado a sugerir que requiera de sus galardonados un juramento de humildad, una especie de juramento hipocrático, que nunca exceda en los pronunciamientos públicos los límites de su competencia (Hayek, 2021).

Una demostración de humildad como sólo es posible ver en los grandes hombres

Se volvió imperativo leer más textos suyos ${ }^{12}$. Me hice la pregunta: ¿Quiénes fueron sus maestros? Es así como lleg otro autor del que vagamente había escuchado habla
udwig von Mises.

Cuando El socialismo - un análisis económico y sociolb

2La bibliografía de Hayek es extensa, pero en mi opinión, además de los textos ya mencionados hay dos obras de él que calificaría de imprescindible lectura: 1) Derecho, legislación y libertad (publicada en tres partes, en los años 1973, 1976 y 1979. En 1982 salió una edicion que unio estas partes en un solo volumen, publicado por Routledge \& Kegan Pauu Lttd); y 2) La fatal arrogancia-los errores del

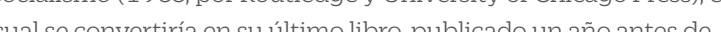$$
\text { . }
$$ 
gico apareció por primera vez en 1922, su impacto fue muy profundo. Alteró gradual, pero fundamentalmente las perspectivas de muchos de los jóvenes idealistas que volvían a sus estudios universitarios después de la [Primera] Guerra Mundial. Lo sé bien, porque yo fui uno de ellos'13 (...) El socialismo prometía satisfacer racional y jeranzas de conseguir un mundo mas racional y justo. Y entonces llegó este libro. Nuestras esperanzas se desvanecieron. El socialismo nos decia dirección equivocada (Hayek, p. 15)

Con estas palabras, el ex miembro de artillería, Hayek, me convenció de leer a Mises para descubrirlo.

En los discursos panfletarios políticos que podian escucharse en la Plaza San Martin, que se ubicaba a pocas cuadras de edificio de mi universidad, se solía decir que el socialis era la solución para los males que aquejaban, desde la ndependencia, al Penc. Usualmente caminaba por ahi, pues a pocos netros libsa hrovista plaza se ubicaban los donde por unas pocas moneds podí llevarme acasa textos de diversos temas que por su antigüedad no podían adquirir en las librerías. Y aunque se corría cierto peligro pasar varias horas en esos puestos ubicados dentro de casonas vetustas sin mantenimiento alguno, era un placer no sólo leer dentro de esas paredes llenas de historias sino también conversar con algunos vendedores que sabía lo que vendían y con otros ocasionales compradores que iniciaban una conversación al darse cuenta de que uno revisaba textos de autores de igual interés para ellos.

Socialismo, tal es el santo y seña de nuestro tiempo. La idea socialista rema hoy dia sobre los espiritus, las masas les son devotas, penetra el pensamiento y sentimiento de todos, eimprime su estilo a nuestra época, que la historia denominará era del socialismo (Mises, 1922/2009, p. 33).

a caída del Muro de Berlin. Hayek muere en marzo de 1992, tres neses después dela disolución dela Union de Repúblicas Socialistas sobre el autodenominado "sccial ismo reat" Havek dijo: "Estuve en una batalla en la que se hablaban once idiomas distintos. Esto tiene que atraer tu atención hacia los problemas de la organización politica" "Ltomado de la página welb de The Mises Institute]. Solo la malaria lo dejó fuera de la guerra. ${ }^{4}$ En el prólogo escrito por Hayek del libro El socialismo (p.15), de

is Forma parte del Centro Historico de Lima, declarado Patrimonio
Con estas palabras, Mises inicia su introducción al libro El socialismo, que vio la luz en 1922 Este mensaie inicial $m$ recordó mucho a la dedicatoria de camino de servidumbre $\mathrm{y}$, sobre todo, a lo que venía pensando desde mis años de estudiante. Lo que escuchaba en la televisión, en la radio. en las conversaciones diarias y en las calles de la vieja Lima se podía resumir en esas primeras líneas.

En esta obra, Mises, quien tambien fue miembro de la artillería austríaca en el frente ruso durante la I Guerra Mundial, hace una crítica al socialismo, concluyendo que es irrealizable, pues "sin propiedad privada de los medios de produccion solo existira a la larga una producción al día para las necesidades personales del individuo" (Mises, 1922/2009, p. 511); y porque el cálculo económico es imposible en el socialismo debido a que "donde no hay mercado no pueden formarse precios, y sin formación de precios no último no existe economía

El socialismo, es un libro que a pesar de haber sido escrito hace cien años ${ }^{16}$ resulta de interés no solo para el politóhace clen años" los diferentes aspectos de la acción humana pues Mises al abordar el gran tema del socialismo toca aspectos tales como el problema sexual, las relaciones entre el hombre la mujer, el matrimonio, el amor libre; asi como el problem que tiene toda persona respecto a la elección de su profesión -dice que en el socialismo no hav una elección espontánea de la carrera-(Mises, 2009, p. 186), y la situación de los artistas, literatos o los que tienen inclinaciones para la ciencia -el socialismo tiene más poder para ejercer presión sobre la libertad intelectual, que cualquier otro emperado que haya existido, nos dice- (Mises, 2009, p. 189). Todo joven de hoy, con interés en que hacer con su vida, puede encontrar luces sobre como una sociedad socialista trata estos tritnos aspectos de manera desventajosa para individuo.

ultural de la Humanidad en 1991 por la UNESCO. Asimismo, L aza san Martm, llamada ass en honor del militar argentino don de su pais Chile y Perí es uno de los lugares $q$ ue ha albergado las manifestaciones politicas más importantes de la historia de este último país.

Hayek, en el prólogo de la obra, estima que Mises la escribio entre 1919 y 1921 (Mises, 1922/2009, p. 18).
Para Mises, el socialismo es destrucción y si algún día llegara a ganar sería mejor calificarlo de "destruccionismo" pues destruye todo lo creado durante siglos por la civilizacion, como el capital. Calificar de manera tan directa y dura a esta ideología en aquellos años en que el socialismo estaba en plena ebullicion por todo el mundo (Lenin aun (lich a URSS) me hizo ver que estaba frente a un intelectual que no se andaba con rodeos; que demostraba valentía de ir como el salmon, contra la corriente. Eso me hizo buscar humana-tratado de economiá, publicado en 1949 .

Pocos libros, como el ya mencionado Camino de servidumbre lo dejan impactado a uno desde su primera página. Con este texto de Mises me ocurrio lo mismo. "Praxeología", un palabra que, hasta antes de abrir ese libro, jamas la habja escuchado. Yla página prinera, que forma parte de su introdución, ll pra por tit

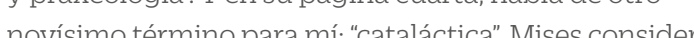

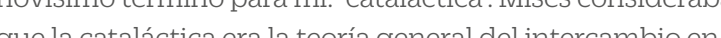
l mercado siendo sus intereses "todos los fenómenos del mercado: surigen su desarrollo asicomo fos consecuencias que provocan" (Mises, 1949/2011 p. 284) que a su vez form parte de la praxeología, la cual es la teoría general de la acción humana (Mises, 1949/2011 p.8.

Para Mises, primero está el pensamiento, luego viene la acción. Esta acción -estudiada por la praxeologia- se distingue entre la acción que es calculable y la que no es susceptible de calcular, siendo la economía una ciencia de aquella parte de la acción humana que, bajo ciertas condiciones, se puede aplicar el cálculo. ¿Y cómo se relaciona el socialismo con la cataláctica? Mises nos dice:

Lo característico del socialismo es que una sola e indivisible voluntad gobierna todas las actividades productivas. Cuando los socialistas aseguran que una economía «ordenada y y «lanificada» reemplazará a "anarquía» de la producción capitalista; que actuaciones racionales sustituiran a la supuesta ausen de lógica del mercado libre, que habra verdadera cooperacion entre los hombres en vez de enconada vez de pra, que se sailistas en definitiva

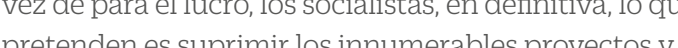
diferentes actuaciones de los consumidores $y$ los de aquellas personas-los empresarios y capitalistasque procuran atender del mejor modo posible los deseos del público, imponiendo en su lugar la exclusiva y monopolística voluntad del jerarca supremo. El socialismo exige la desaparición del mercado y de la competencia cataláctica17 . El sistema es incompatible con el mercado, con los precios y con la competencia, pues pone todos los resortes economicos en manos de Una unica autoridad (Mises, 1949/2011, p. 832)

Estudiar la economía enmarcándola en una filosofía de la accion humana me resultó no solamente muy novedoso. sino tambien muy provechoso, pues le da sentido al por qué actuamos como lo hacemos en el mercado, y cómo la libertad de actuar dentro de este ha beneficiado a todas aquellas sociedades que han permitido ello. El aporte de Mises al entendimiento de la economía es invaluable, y de la libertad en nuestras sociedndes Acera de este the probablemente más que de ningín 2001, p. 11).

Desde muy niño siempre me acercaba con cierto sigilo a la copiosa biblioteca de mi padre Aunque án no sabí leer, me gustaba abrir los libros y curiosear sus páginas y si estas tenían dibujos o pinturas, pues mucho mejor. Uno de aquellos libros era sobre Copérnico ${ }^{20}$, pues me llamaba la atención su retrato que ocupaba toda la portada y las. páginas interiores llenas de fotografías de los edificios. calles y paisajes relacionados con la vida de este sabio monje que, además de astrónomo y matemático fue, entre otras cosas, economista. Ya en la escuela pude enterarme que habia sido el autor de la teoria heliocentrica, poniendo las cosas en su lugar en el universo: los cuerpos celestes no giran alrededor de la Tierra, sino estos (incluso la Tierra) Io hacen alrededor del Sol.

Una sección muy grande de la biblioteca de mi padre correspondía a la economí pues él enseñaba esta mater

${ }^{17}$ Las cursivas son mías

- Subibliografia es también extensa. Pero aqui menciono dos cros de el que llamaron mi atención:1) Nación, estado y economia (1919) y,2) Liberalismo-la tradición clásica (1927).

. Margit, en 1940, pero viol laluz en 1978 , debidolo a que pidió que no sea 20 El libro es Nicolás Copérnico y suépoca de Jan Adamczewski (1972) Sébien los datos ahora pues el libro lo heredéde mi padre hora se encuentra en mi biblioteca personal. 
en la Universidad Nacional de Ingeniería (UNI)212. En ellos siempre veía números operaciones matemáticas, fórmulas diagramas y curvas Y en mis clases universitarias de economía, la realidad era la misma. ¿Tiene que ser la economía siempre explicada con números? Un día Mises me dijo en uno de sus libros: "En torno a la Navidad de 1903, lei por primera vez los Grundzätze der Volkswirts chaftslehre ${ }^{22}$ de Menger, y fue esta lectura la que hizo que me convirtiera en economista"23 (Mises, 1949/2011, p. 67).

Nacido en febrero de 1840 en Neu-Sandec (en ese entonces perteneciente al Imperio Austrohúngaro y hoy ubicada en erritonio polaco), Can Menger es considerado el fundador de Ia Escuela Austriaca de Economía (Mises y Hayek, en realidad, pertenecen a la segunda generación). La característica comun de todos los partidarios de la Escuela Austriaca, lo que les contirio su peculiaridad e hizo posibles sus poste-

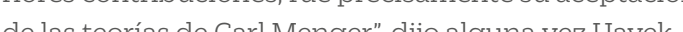
(1992/1996, p. 68).

Recordaba la teoría marginalista de mis clases universitarias pero no de aquel nombre Menger era un apellido nuevo pero no de aquel nombre. Menger era un apellido nuevo nunca lo mencionaron Hayek luego me alivió cuando me

Pero difícilmente se encontrará en esta historia [de la economía política], ni en la de ninguna otra rama del saber, el ejemplo de un autor que hava revolucionado los fundamentos de una ciencia ya bien establecida y haya conseguido por ello general reconocimiento y que, a pesar de todo, haya sido tan desconocido como Carl Menger (Hayek, 1992/1996, p. 68)

Y fue Menger quien me permitió entender la esencia de la economia sin necesidad de usar las matemáticas Había llegado al fundador de una escuela de economia sin proponèrmelo. Solo segui el hilo que se inició con la lectura del ibro deunator al cuat ligué por la simple

Aunque creada en 1955 como universidad, su existencia viene

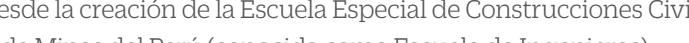
fundada en 1876 por Eduardo I de Habich ingeniero polaco (la ciudad natal de Copérnico hoy pertenece a Polonia) 22 Principios de economía politica (1871). ${ }^{3}$ En Autobiografía de un liberal-La Gran Viena contra el estatalismo por uno de mis autores favoritos de ciencia política. Había entrado a un universo totalmente desconocido para mí y. sin embargo, solo había atisbado un sistema solar dentro de una galaxia mayor. Los miembros de la Escuela Austriaca de Economia no sólo hablaban de esta materia, sino asicología y demás diosifia, derecho, ciencias sociales, ue todo eso, ellos tenian undenominador común: defendía a libertad del individuo. Y no estaban tan solos.

El libro acerca de Copérnico, el cual llamó mi atención desde mi primera infancia, culmina con estas palabras: con los ojos ifjos en el fimanento estrellado transformó el orden del mundo en que le correspondio vivir, y al nuestro (Dabno el canino de las estrellas (Adamezewski, 1972 p.149.) Hayek conthbuyb enomemente para que no pidasemos aquel mandoque creó hsticuciones para

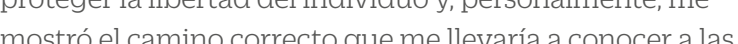
.

\section{REFERENCIAS}

Adamczewski, J. (1972). Nicolás Copérnico y suépoca Varsovia, Polonia: Ediciones Interpress.

Eolegio de la Inmaculada, Jesuitas - Lima. (s.I.). Reseña histórica. https://ci.edu.pe/acerca/\#resena

Free To Choose Network. (2016). Friedrich von Hayek and James Buchanan Part I (S1029) - Full Video [Video], YouTube. https://ww mod ses\&t=2267s

Hayek, F. A. (2017). Camino de servidumbre. Madrid, España: Unión Editorial (Obra original publicada en 1944)

Hayek, F. A. (1996). Las vicisitudes del liberalismo. Ensayos sobre economía austriaca y el ideal de libertad. Madrid, España: Unión Editorial (Obra origina publicada en 1992)

Hayek, F.A. (2014) Los fundamentos de la libertad Madrid, España: Unión Editorial (Obra original publicada
Hayek, F. A. (2015). Nuevos estudios de filosofía, politica economía e historia de las ideas. Madrid, España:

. [Transcripción del discurso en el banquete Nobel, 10 de diciembre de 1974] Nobel Media AB. Recuperado de https://www.nobelprize.org/prizes/

economic-sciences/1974/hayek/speech/

McCaffrey, M. (2018, 6 de noviembre). Los economistas austriacos en la Primera Guerra Mundial [Publicación web]. Mises Institute. Recuperado de https://mises. org/es/wire/los-economistas-austriacos-en-la-

Mises, L. (2011). La acción humana. Tratado de economía. Madrid, España: Unión Editorial (Obra original publicada en 1949)

Mises. L. (2009). El socialismo. Análisis económico y sociológico. Madrid. España: Unión Editorial (Obra original publicada en 1922)

Mises, L. (2001). Autobiografía de un liberal - La Gran Viena contra el estatalismo. Madrid: Unión Editorial

Sartori, G. (2000). Teoría de la democracia. Madrid, España Alianza Editorial. (Obra original publicada en 1987) 